\title{
2
}

\section{Delay Stabilization of Rotating Waves Without Odd Number Limitation}

\author{
B. Fiedler, V. Flunkert, M. Georgi, P. Hövel, and E. Schöll
}

\section{1 \\ Introduction}

A variety of methods have been developed in nonlinear science to stabilize unstable periodic orbits (UPOs) and control chaos [1], following the seminal work by Ott, Grebogi and Yorke [2], who employed a tiny control force to stabilize UPOs embedded in a chaotic attractor $[3,4]$. A particularly simple and efficient scheme is time-delayed feedback as suggested by Pyragas [5], which uses the difference $z(t-\tau)-z(t)$ of a signal $z$ at a time $t$ and a delayed time $t-\tau$. It is an attempt to stabilize periodic orbits of (minimal) period $T$ by a feedback control which involves a time delay $\tau=n T$, for suitable positive integer $n$. A linear feedback example is

$$
\dot{z}(t)=f(\lambda, z(t))+B[z(t-\tau)-z(t)]
$$

where $\dot{z}(t)=f(\lambda, z(t))$ describes a d-dimensional nonlinear dynamical system with bifurcation parameter $\lambda$ and an unstable orbit of (minimal) period $T$. The constant feedback control matrix $B$ is chosen suitably. Typical choices are multiples of the identity or of rotations, or matrices of low rank. More general nonlinear feedbacks are conceivable, of course. The main point, however, is that the Pyragas choice $\tau_{P}=n T$ of the delay time eliminates the feedback term on the orbit, and thus recovers the original $T$-periodic solution $z(t)$. In this sense the method is noninvasive.

Although time-delayed feedback control has been widely used with great success in real world problems in physics, chemistry, biology, and medicine, e.g. [6-19], a severe limitation used to be imposed by the common belief that certain orbits cannot be stabilized for any strength of the control force. In fact, it had been contended that periodic orbits with an odd number of real Floquet multipliers greater than unity cannot be stabilized by the Pyragas method [20-25], even if the simple scheme (2.1) is extended by multiple delays in form of an infinite series [26]. To circumvent this restriction more complicated control schemes, like an oscillating feedback [27], half-period delays for special, symmetric orbits [28], or the introduction of an additional, unstable 
degree of freedom [25,29], have been proposed. Recently, however, it has been shown that the general limitation for orbits with an odd number of real unstable Floquet multipliers greater than unity does not hold: stabilization may be possible for suitable choices of $B[30,31]$. As an example, an unstable periodic orbit generated by a subcritical Hopf bifurcation has been considered. In particular, this refutes the theorem in [21]. In this article we review these recent findings, and give some general symmetry and stability considerations.

\section{2}

\section{Mechanism of Stabilization}

Consider the normal form of a subcritical Hopf bifurcation, extended by a time-delayed feedback term

$$
\dot{z}(t)=\left[\lambda+i+(1+i \gamma)|z(t)|^{2}\right] z(t)+b[z(t-\tau)-z(t)]
$$

with $z \in \mathbb{C}$ and real parameters $\lambda$ and $\gamma$. Here the Hopf frequency is normalized to unity. The feedback matrix $B$ is represented by multiplication with a complex number $b=b_{R}+i b_{I}=b_{0} e^{i \beta}$ with real $b_{R}, b_{I}, \beta$, and positive $b_{0}$. Note that the nonlinearity $f(\lambda, z(t))=\left[\lambda+i+(1+i \gamma)|z(t)|^{2}\right] z(t)$ and the control $B=b$ commute with complex rotations. Therefore $\exp (i \vartheta) z(t)$ solves Eq. (2.2), for any fixed $\vartheta$, whenever $z(t)$ does. In particular, nonresonant Hopf bifurcations from the trivial solution $z \equiv 0$ at simple imaginary eigenvalues $\eta=i \omega \neq 0$ produce rotating wave solutions $z(t)=z(0) \exp \left(i \frac{2 \pi}{T} t\right)$ with minimal period $T=2 \pi / \omega$ even in the nonlinear case and with delay terms. This follows from uniqueness of the emanating Hopf branches [32].

Transforming Eq. (2.2) to amplitude and phase variables $r(t), \varphi(t)$ for $z(t)=$ $r(t) e^{i \varphi(t)}$, we obtain at vanishing control $b=0$

$$
\begin{aligned}
& \dot{r}=\left(\lambda+r^{2}\right) r \\
& \dot{\varphi}=1+\gamma r^{2} .
\end{aligned}
$$

An unstable periodic orbit (UPO) with $r=\sqrt{-\lambda}$ and period $T=2 \pi /(1-\gamma \lambda)$ exists for $\lambda<0$. This is the orbit which we will stabilize, called the Pyragas orbit. At $\lambda=0$ a subcritical Hopf bifurcation occurs, and the steady state $z=0$ loses its stability. The Pyragas control method is a noninvasive method: the control force vanishes at successful stabilization, and the periodic Pyragas orbit itself remains untouched by the control procedure. In order to satisfy this requirement the delays $\tau$ have to be chosen as a multiple of the minimal period: $\tau=n T$. This defines the local Pyragas curve in the $(\lambda, \tau)$-plane for any $n \in \mathbb{N}$

$$
\tau_{P}(\lambda)=\frac{2 \pi n}{1-\gamma \lambda}=2 \pi n(1+\gamma \lambda+\ldots),
$$


emanating from the Hopf bifurcation points $\lambda=0, \tau=2 \pi n$, towards negative $\lambda$.

Under further nondegeneracy conditions, the Hopf point $\lambda=0, \tau=n T$ $\left(n \in \mathbb{N}_{0}\right.$ ) also continues to a Hopf bifurcation curve $\tau=\tau_{H}(\lambda)$ for $\lambda<0$. We determine this Hopf curve next. It is characterized by purely imaginary eigenvalues $\eta=i \omega$ of the transcendental characteristic equation

$$
\eta=\lambda+i+b\left(e^{-\eta \tau}-1\right)
$$

which results from the linearization at the steady state $z=0$ of the delayed system Eq. (2.2). Separating Eq. (2.6) into real and imaginary parts

$$
\begin{aligned}
0 & =\lambda+b_{0}[\cos (\beta-\omega \tau)-\cos \beta] \\
\omega-1 & =b_{0}[\sin (\beta-\omega \tau)-\sin \beta]
\end{aligned}
$$

and using the trigonometric identity

$$
[\cos (\beta-\omega \tau)]^{2}+[\sin (\beta-\omega \tau)]^{2}=1
$$

to eliminate $\omega(\lambda)$ from Eqs. (2.7) and (2.8) yields an explicit expression for the multivalued Hopf curve $\tau_{H}(\lambda)$ for given control amplitude $b_{0}$ and phase $\beta$ :

$$
\tau_{H}=\frac{ \pm \arccos \left(\frac{b_{0} \cos \beta-\lambda}{b_{0}}\right)+\beta+2 \pi n}{1-b_{0} \sin \beta \mp \sqrt{\lambda\left(2 b_{0} \cos \beta-\lambda\right)+b_{0}^{2} \sin ^{2} \beta}} .
$$

Note that $\tau_{H}$ is not defined in case of $\beta=0$ and $\lambda<0$. Thus complex $b$ is a necessary condition for the existence of the Hopf curve in the subcritical regime $\lambda<0$. Figure 2.1 jointly displays the family of Hopf curves $n=0,1, \ldots$ (solid), Eq. (2.10), and the Pyragas curve $n=1$ (dashed), Eq. (2.5), in the $(\lambda, \tau)$ plane. In Fig. 2.1(b) the domains of instability of the trivial steady state $z=0$, bounded by the Hopf curves, are marked by light grey shading. The dimensions of the unstable manifold of $z=0$ are given in parentheses along the $\tau$-axis in Fig. 2.1(b). By construction, the delay $\tau$ becomes a multiple of the minimal period $T$ of the bifurcating Pyragas orbits along the Pyragas curve $\tau=\tau_{p}(\lambda)=n T$, and the time-delayed feedback term vanishes on these periodic orbits, noninvasively.

Standard exchange of stability results [32], which hold verbatim for delay equations, then assert that the bifurcating branch of periodic solutions locally inherits linear asymptotic (in)stability from the trivial steady state, i.e., it consists of stable periodic orbits on the Pyragas curve $\tau_{P}(\lambda)$ inside the shaded domains for small $|\lambda|$. We stress that an unstable trivial steady state is not a sufficient condition for stabilization of the Pyragas orbit. In fact, the stabilized Pyragas orbit can destabilize again when $\lambda<0$ is decreased further. This may 
(a)

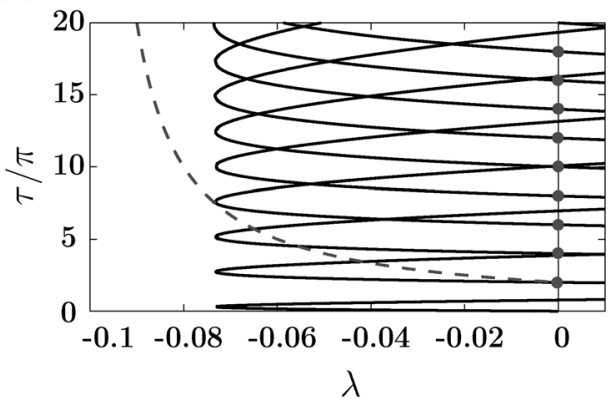

(b)

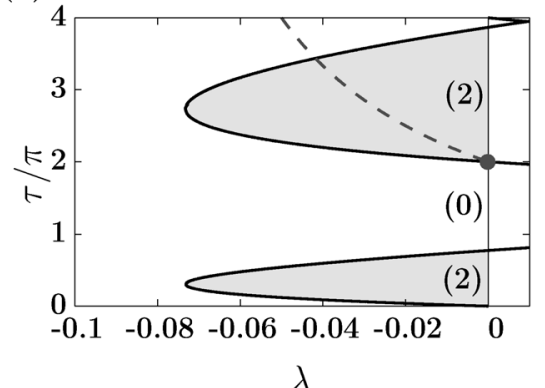

Fig. 2.1 Pyragas (dashed) and Hopf (solid) curves in the $(\lambda, \tau)$ plane: (a) Hopf bifurcation curves $n=0, \ldots, 10$, (b) Hopf bifurcation curves $n=0,1$ in an enlarged scale. Light grey shading marks the domains of unstable $z=0$ and numbers in parentheses denote the dimension of the unstable manifold of $z=0(\gamma=-10$, $b_{0}=0.3$ and $\left.\beta=\pi / 4\right)$.

be caused, for instance, by a torus bifurcation, see Section 2.5. However, there exists an interval of $\lambda<0$ in our example for which Pyragas stabilization holds.

More precisely, periodic orbits of the controlled and uncontrolled system, alike, possess a unique and simple nontrivial Floquet multiplier $\mu=\exp (\Lambda T)$, near unity, as long as $|\lambda|$ remains small. All other nontrivial Floquet multipliers lie strictly inside the complex unit circle. In particular, the (strong) unstable dimension of these periodic orbits is either 0 or 1 , depending on $\mu<1$ or $\mu>1$, respectively, and their unstable manifold is absent or two-dimensional. This is shown in Fig. 2.2 panel (a) top, which depicts solutions $\Lambda$ of the characteristic equation of the periodic solution on the Pyragas curve (see Appendix).

The largest real part is positive for $b_{0}=0$. Thus the periodic orbit of the uncontrolled system is born unstable. As the amplitude of the feedback gain increases, the largest real part of the eigenvalue becomes smaller and eventually changes sign at TC. Hence the periodic orbit is stabilized. Note that an infinite number of Floquet exponents are created by the control scheme; their real parts tend to $-\infty$ in the limit $b_{0} \rightarrow 0$ of vanishing control. Some of them may cross over to positive real parts for larger $b_{0}$ (dashed line in Fig. 2.2a), terminating the stability of the periodic orbit; see Section 2.5. Panel (a) bottom illustrates the stability of the steady state by displaying the largest real part of the eigenvalues $\eta$.

Figure 2.2 (b) shows the behavior of the Floquet multipliers $\mu=\exp (\Lambda T)$ of the Pyragas orbit in the complex plane with increasing amplitude of the feedback gain $b_{0}$ as a parameter (marked by arrows). There is an isolated real multiplier crossing the unit circle at $\mu=1$, in contrast to the result stated in [21]. 

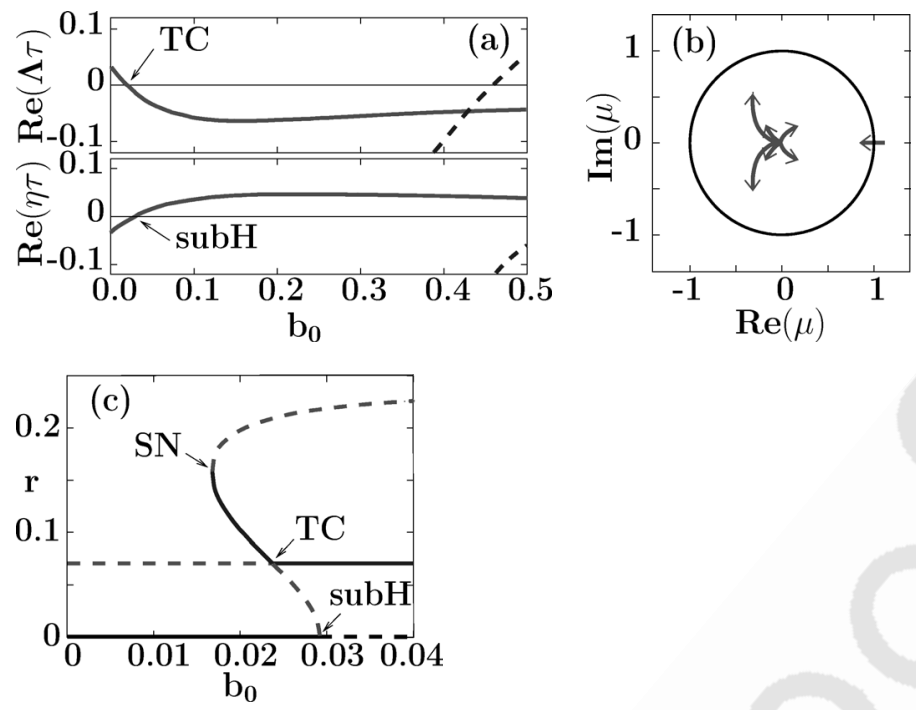

Fig. 2.2 (Color online) (a) top: Real part of Floquet exponents $\Lambda$ of the periodic orbit vs. feedback amplitude $b_{0}$. bottom: Real part of eigenvalue $\eta$ of steady state vs. feedback amplitude $b_{0}$. (b) Floquet multipliers $\mu=\exp (\Lambda T)$ (red) in the complex plane with the feedback amplitude $b_{0} \in[0,0.3]$ as a parameter. (c) Radii of periodic orbits. Solid (dashed) lines correspond to stable (unstable) orbits. $\left(\lambda=-0.005, \gamma=-10, \tau=\frac{2 \pi}{1-\gamma \lambda}, \beta=\pi / 4\right)$.

This is caused by a transcritical bifurcation at which the Pyragas orbit collides with a delay-induced stable periodic orbit. In panel (c) of Fig. 2.2 the radii of all circular periodic orbits $(r=$ const $)$ are plotted versus the feedback strength $b_{0}$. For small $b_{0}$ only the initial (unstable) Pyragas orbit ( $T$ and $r$ independent of $b_{0}$ ) and the steady state $r=0$ (stable) exist. With increasing $b_{0}$ a pair of unstable/stable periodic orbits is created in a saddle-node (SN) bifurcation. The stable one of the two orbits (solid) then exchanges stability with the Pyragas orbit in a transcritical bifurcation (TC), and terminates at in a subcritical Hopf bifurcation (subH), where the steady state $r=0$ becomes unstable. The Pyragas orbit continues as a stable periodic orbit for larger $b_{0}$. Except at TC, the delay-induced orbit has a period $T \neq \tau$. Note that the respective exchanges of stability of the Pyragas orbit (TC) and the steady state (subH) occur at slightly different values of $b_{0}$. This is also corroborated by Fig. 2.2(c). The mechanism of stabilization of the Pyragas orbit by a transcritical bifurcation relies upon the possible existence of such delay-induced periodic orbits with $T \neq \tau$. Technically, the proof of the odd-number limitation theorem in [21] fails because the trivial Floquet multiplier $\mu=1$ (Goldstone mode of periodic orbit) was neglected there; $F(1)$ in Eq. (14) in [21] is thus zero and not less than zero, as 
assumed [33]. At TC, where a second Floquet multiplier crosses the unit circle, this results in a Floquet multiplier $\mu=1$ of algebraic multiplicity two.

\section{3}

\section{$S^{1}$-Symmetry and Stability of Rotating Waves}

For any fixed real $\omega$, the transformation $z(t)=e^{i \omega t} \zeta(t)$ to co-rotating complex coordinates $\zeta(t)$ transforms Eq. (2.2) into the equivalent delay equation

$$
\begin{aligned}
\dot{\zeta}(t) & =(\lambda+(1-\omega) i) \zeta(t)+(1+i \gamma)|\zeta(t)|^{2} \zeta(t) \\
& -b_{0} \exp (i \beta)\left(\zeta(t)-e^{-i \omega \tau} \zeta(t-\tau)\right) .
\end{aligned}
$$

The co-rotating equation (2.11) remains autonomous since $e^{i \vartheta} z(t)$ solves Eq. (2.2) for any fixed real $\vartheta$, whenever $z(t)$ does. Steady states $\dot{\zeta}=0$ of Eq. (2.11) are precisely the rotating waves of Eq. (2.2), i.e., solutions of the form $z(t)=e^{i \omega t} \zeta_{0}$ with nonzero rotation frequency $\omega$ and nonzero $\zeta_{0} \in \mathbb{C}$. The minimal period of such solutions $z(t)$ is of course given by $T=2 \pi /|\omega|$.

For $\tau=n T=2 \pi n /|\omega|$ with $n=1,2,3, \ldots$ we obtain the non-invasive Pyragas control and the Pyragas curves (2.5).

Rotating waves, alias nontrivial steady states $\zeta(t)=\zeta_{0}$ of Eq. (2.11), are solutions of

$$
0=\lambda+(1-\omega) i+(1+i \gamma)\left|\zeta_{0}\right|^{2}-b_{0} \exp (i \beta)\left(1-e^{-i \omega \tau}\right) .
$$

The bifurcation diagram in Fig 2.2 (c) displays solutions $(\omega, r), r=\left|\zeta_{0}\right|$, of Eq. (2.12) only. We can solve this equation for real parts

$$
\left|\zeta_{0}\right|^{2}=-\lambda-b_{0}[\cos (\beta-\omega \tau)-\cos (\beta)]
$$

under the constraint of a positive right hand side. Substituting into the imaginary part of Eq. (2.12) yields the real equation

$$
\begin{aligned}
0 & =1-\omega+b_{0}[\sin (\beta-\omega \tau)-\sin (\beta)] \\
& -\gamma\left\{\lambda+b_{0}[\cos (\beta-\omega \tau)-\cos (\beta)]\right\} .
\end{aligned}
$$

We seek solutions $\omega$, depending on the five real parameters $\lambda, \gamma, b_{0}, \beta, \tau$. The degenerate case $\omega=0$ which corresponds to a circle of equilibria, alias a frozen wave of vanishing angular velocity $\omega$, arises only for $\gamma \lambda=1$ [34], i.e., far away from the Hopf bifurcation.

The stabilization mechanism outlined in Section 2.2 can be understood from a mathematical view point as follows. At Hopf bifurcation we have a simple pair of purely imaginary eigenvalues, and no other imaginary eigenvalues. 
Therefore, the center manifold is two-dimensional at Hopf bifurcation [32]. Dimension two extends and includes the nearby transcritical bifurcation of rotating waves. Moreover, the center manifold can be chosen to be invariant with respect to the $S^{1}$ action $z \mapsto e^{i \vartheta} z$ [35]. In polar coordinates $z=r e^{i \varphi}$ the dynamics in any two-dimensional center manifold is therefore given by a system of the general form

$$
\begin{aligned}
& \dot{r}=f\left(r^{2}, \underline{\mu}\right) r \\
& \dot{\varphi}=g\left(r^{2}, \underline{\mu}\right)
\end{aligned}
$$

with parameter vector $\mu$, i.e., in our case $\mu=\left(\lambda, \gamma, b_{0}, \beta, \tau\right)$. Note that $\varphi$ neither enters the equation for $\dot{r}$ nor for $\dot{\varphi}$. Indeed, $(r(t), \varphi(t)+\vartheta)$ must be a solution for any fixed $\vartheta$, by $S^{1}$-equivariance, whenever $(r(t), \varphi(t))$ is. Also note that Eq. (2.15a) is a system of differential equations which does not involve time delayed arguments. Rather, the original time delay $\tau$ enters as one parameter among others.

To determine $f$ on the center manifold, we first observe that $f\left(r^{2}, \mu\right)=0$ defines rotating (or frozen) waves with $\left|\zeta_{0}\right|=r$, and thus must be equivalent to Eq. (2.12) with $\omega=g\left(r^{2}, \mu\right)$. Conversely, the solution set $\left(r^{2}, \omega, \mu\right)$ is therefore given by Eqs. (2.13) and (2.14), and defines the zero set of $f$. Again, $f\left(r^{2}, \mu\right)=0$ if, and only if, $(\omega, \mu)$ solve Eq. (2.14) and $r^{2}$ is given by Eq. (2.13).

To determine the stability of our rotating waves within the center manifold it remains to determine the sign of $f$ outside the zero set. That sign is known at the trivial equilibrium $r=0$, by standard exchange of stability at nondegenerate Hopf bifurcations. Normally hyperbolic rotating waves correspond to simple zeros of $f$ in the $r$-direction, i.e., $\partial_{r} f \neq 0$. This allows us to determine the sign of $f$ in the bifurcation diagram Fig. 2.2 (c). The (in-)stability properties of all rotating waves within the two-dimensional center manifold are then immediate. Spectral analysis at the Hopf bifurcation shows strict stability of the remaining nonimaginary eigenvalues. Therefore the center manifold is attractive [32]. In particular, (in-)stability of rotating waves in the full delay system (2.2) is inherited from the center manifold analysis without ever computing the manifold itself. In conclusion this proves the stability properties indicated in Fig. 2.2 (c), for the parameters chosen there.

\section{4}

\section{Conditions on the Feedback Gain}

Next we analyse the conditions under which stabilization of the subcritical periodic orbit is possible. From Fig. 2.1(b) it is evident that the Pyragas curve must lie inside the yellow region, i.e., the Pyragas and Hopf curves emanating from the point $(\lambda, \tau)=(0,2 \pi)$ must locally satisfy the inequality $\tau_{H}(\lambda)<$ 
$\tau_{P}(\lambda)$ for $\lambda<0$. More generally, let us investigate the eigenvalue crossings of the Hopf eigenvalues $\eta=i \omega$ along the $\tau$-axis of Fig. 2.1. In particular we derive conditions for the unstable dimensions of the trivial steady state near the Hopf bifurcation point $\lambda=0$ in our model equation (2.2). On the $\tau$-axis $(\lambda=0)$, the characteristic equation (2.6) for $\eta=i \omega$ is reduced to

$$
\eta=i+b\left(e^{-\eta \tau}-1\right),
$$

and we obtain two series of Hopf points given by

$$
\begin{aligned}
& 0 \leq \tau_{n}^{A}=2 \pi n \\
& 0<\tau_{n}^{B}=\frac{2 \beta+2 \pi n}{1-2 b_{0} \sin \beta} \quad(n=0,1,2, \ldots) .
\end{aligned}
$$

The corresponding Hopf frequencies are $\omega^{A}=1$ and $\omega^{B}=1-2 b_{0} \sin \beta$, respectively. Note that series A consists of all Pyragas points, since $\tau_{n}^{A}=$ $n T=\frac{2 \pi n}{\omega^{A}}$. In the series $B$ the integers $n$ have to be chosen such that the delay $\tau_{n}^{B} \geq 0$. The case $b_{0} \sin \beta=1 / 2$, only, corresponds to $\omega^{B}=0$ and does not occur for finite delays $\tau$.

We evaluate the crossing directions of the critical Hopf eigenvalues next, along the positive $\tau$-axis and for both series. Abbreviating $\frac{\partial}{\partial \tau} \eta$ by $\eta \tau$ the crossing direction is given by $\operatorname{sign}\left(\operatorname{Re} \eta_{\tau}\right)$. Implicit differentiation of Eq. (2.16) with respect to $\tau$ at $\eta=i \omega$ implies

$$
\operatorname{sign}\left(\operatorname{Re} \eta_{\tau}\right)=-\operatorname{sign}(\omega) \operatorname{sign}(\sin (\omega \tau-\beta)) .
$$

We are interested specifically in the Pyragas-Hopf points of series A (marked by dots in Fig. 2.1) where $\tau=\tau_{n}^{A}=2 \pi n$ and $\omega=\omega^{A}=1$. Indeed $\operatorname{sign}\left(\operatorname{Re} \eta_{\tau}\right)=\operatorname{sign}(\sin \beta)>0$ holds, provided we assume $0<\beta<\pi$, i.e., $b_{I}>0$ for the feedback gain. This condition alone, however, is not sufficient to guarantee stability of the steady state for $\tau<2 n \pi$. We also have to consider the crossing direction $\operatorname{sign}\left(\operatorname{Re} \eta_{\tau}\right)$ along series $\mathrm{B}, \omega^{B}=1-2 b_{0} \sin \beta$, $\omega^{B} \tau_{n}^{B}=2 \beta+2 \pi n$, for $0<\beta<\pi$. Equation (2.19) now implies $\operatorname{sign}\left(\operatorname{Re} \eta_{\tau}\right)=$ $\operatorname{sign}\left(\left(2 b_{0} \sin \beta-1\right) \sin \beta\right)=\operatorname{sign}\left(2 b_{0} \sin \beta-1\right)$.

To compensate for the destabilization of $z=0$ upon each crossing of any point $\tau_{n}^{A}=2 \pi n$, we must require stabilization $\left(\operatorname{sign}\left(\operatorname{Re} \eta_{\tau}\right)<0\right)$ at each point $\tau_{n}^{B}$ of series B. If $b_{0} \geq 1 / 2$, this requires $0<\beta<\arcsin \left(1 /\left(2 b_{0}\right)\right)$ or $\pi-$ $\arcsin \left(1 /\left(2 b_{0}\right)\right)<\beta<\pi$. The distance between two successive points $\tau_{n}^{B}$ and $\tau_{n+1}^{B}$ is $2 \pi / \omega^{B}>2 \pi$. Therefore, there is at most one $\tau_{n}^{B}$ between any two successive Hopf points of series A. Stabilization requires exactly one such $\tau_{n}^{B}$, specifically: $\tau_{k-1}^{A}<\tau_{k-1}^{B}<\tau_{k}^{A}$ for all $k=1,2, \ldots, n$. This condition is satisfied if, and only if,

$$
0<\beta<\beta_{n}^{*}
$$


where $0<\beta_{n}^{*}<\pi$ is the unique solution of the transcendental equation

$$
\frac{1}{\pi} \beta_{n}^{*}+2 n b_{0} \sin \beta_{n}^{*}=1 .
$$

This holds because the condition $\tau_{k-1}^{A}<\tau_{k-1}^{B}<\tau_{k}^{A}$ first fails when $\tau_{k-1}^{B}=\tau_{k}^{A}$. Equation (2.20) represents a necessary but not yet sufficient condition that the Pyragas choice $\tau_{P}=n T$ for the delay time will stabilize the periodic orbit.

To evaluate the remaining condition, $\tau_{H}<\tau_{P}$ near $(\lambda, \tau)=(0,2 \pi)$, we expand the exponential in the characteristic equation (2.6) for $\omega \tau \approx 2 \pi n$, and obtain the approximate Hopf curve for small $|\lambda|$ :

$$
\tau_{H}(\lambda) \approx 2 \pi n-\frac{1}{b_{I}}\left(2 \pi n b_{R}+1\right) \lambda .
$$

Recalling (2.5), the Pyragas stabilization condition $\tau_{H}(\lambda)<\tau_{P}(\lambda)$ is therefore satisfied for $\lambda<0$ if, and only if,

$$
\frac{1}{b_{I}}\left(b_{R}+\frac{1}{2 \pi n}\right)<-\gamma .
$$

Equation (2.23) defines a domain in the plane of the complex feedback gain $b=b_{R}+i b_{I}=b_{0} e^{i \beta}$ bounded from below (for $\gamma<0<b_{I}$ ) by the straight line

$$
b_{I}=\frac{1}{-\gamma}\left(b_{R}+\frac{1}{2 \pi n}\right) \text {. }
$$

Equation (2.21) represents a curve $b_{0}(\beta)$, i.e.,

$$
b_{0}=\frac{1}{2 n \sin \beta}\left(1-\frac{\beta}{\pi}\right) \text {, }
$$

which forms the upper boundary of a domain given by the inequality (2.20). Thus Eqs. (2.24) and (2.25) describe the boundaries of the domain of control in the complex plane of the feedback gain $b$ in the limit of small $\lambda$. Figure 2.3 depicts this domain of control for $n=1$, i.e., a time delay $\tau=\frac{2 \pi}{1-\gamma \lambda}$. The lower and upper solid curves correspond to Eq. (2.24) and (2.25), respectively. The grayscale displays the numerical result of the largest real part, wherever $<0$, of the Floquet exponent, calculated from linearization of the amplitude and phase equations around the periodic orbit (see Appendix). Outside the shaded areas the periodic orbit is not stabilized. With increasing $|\lambda|$ the domain of stabilization shrinks, as the deviations from the linear approximation (2.22) become larger. For sufficiently large $|\lambda|$ stabilization is no longer possible, in agreement with Fig. 2.1(b). Note that for real values of $b$, i.e., $\beta=0$, no stabilization occurs at all. Hence, stabilization fails if the feedback matrix $B$ is a multiple of the identity matrix. 


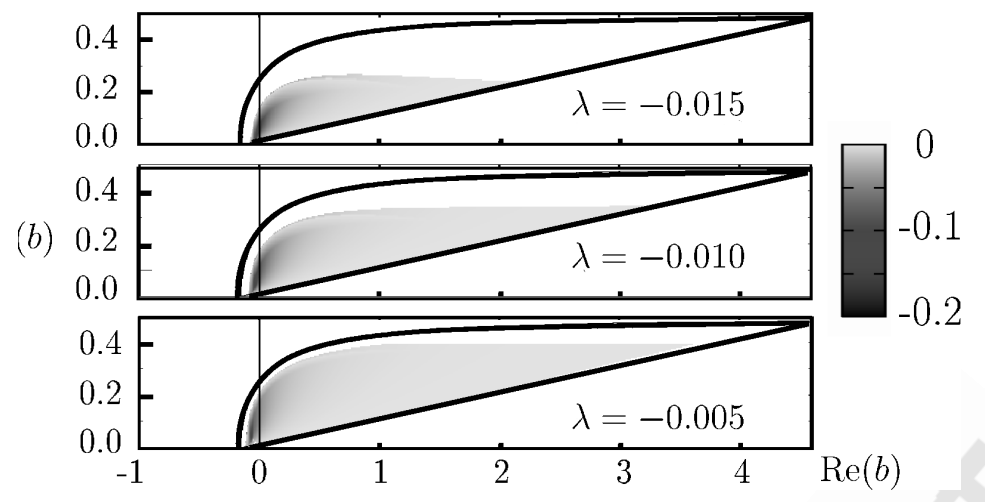

Fig. 2.3 Domain of control in the plane of the complex feedback gain $b=b_{0} e^{i \beta}$ for three different values of the bifurcation parameter $\lambda$. The solid curves indicate the boundary of stability in the limit $\lambda \nearrow 0$, see Eqs. (2.24) and (2.25). The shading shows the magnitude of the largest (negative) real part of the Floquet exponents of the periodic orbit $\left(\gamma=-10, \tau=\frac{2 \pi}{1-\gamma \lambda}\right)$.

\section{5}

Tori

Rotating waves $z(t)=e^{i \omega t} \zeta_{0}$ are periodic solutions of Eq. (2.2). But not all periodic solutions need to be rotating waves. Any bifurcation from rotating waves, however, must be visible in co-rotating coordinates $\zeta(t)=e^{-i \omega t} z(t)$ as well. Since rotating waves of Eq. (2.2) are equilibria of Eq. (2.11) any such bifurcation must be accompanied by purely imaginary eigenvalues $\Lambda$ of the characteristic equation associated to $\zeta_{0}$.

At the rotating wave equilibrium $r_{0}=\zeta_{0}>0$ of the co-rotating system Eq. (2.11), the characteristic equation for Floquet multipliers $\mu=e^{\Lambda T}$ becomes

$$
0=\chi(\Lambda)=\Lambda^{2}-2\left(r_{0}^{2}+b_{0} c E\right) \Lambda+2 r_{0}^{2}(c+\gamma s) b_{0} E+\left(b_{0} E\right)^{2} .
$$

See the appendix for a derivation. Here we have abbreviated $c=\cos \beta, s=$ $\sin \beta$ for the control $b=b_{0} e^{\beta}$, and $E=e^{-\Lambda \tau}-1$ for the Floquet exponents $\Lambda$.

In Fig. 2.2 (a) we have indicated how the real part $\operatorname{Re} \Lambda$ of a delay induced Floquet exponent of the Pyragas orbit $z(t)$ crosses zero when the control amplitude $b_{0}$ is increased (dashed line). In Figs. 2.4 and 2.5 we indicate the resulting dynamic consequences for a particular choice of parameters.

The observed amplitude oscillations can be explained by a supercritical Neimark-Sacker torus bifurcation scenario. Indeed the nontrivial purely imaginary Floquet exponent $\Lambda$ causes the associated nonreal Floquet multiplier $\mu=e^{\Lambda T}$ to cross the complex unit circle and destabilize the periodic Pyragas orbit inspite of an increasing control amplitude (see Fig. 2.4). The resulting bifurcation is supercritical in our example; see also Fig. 2.5. The 


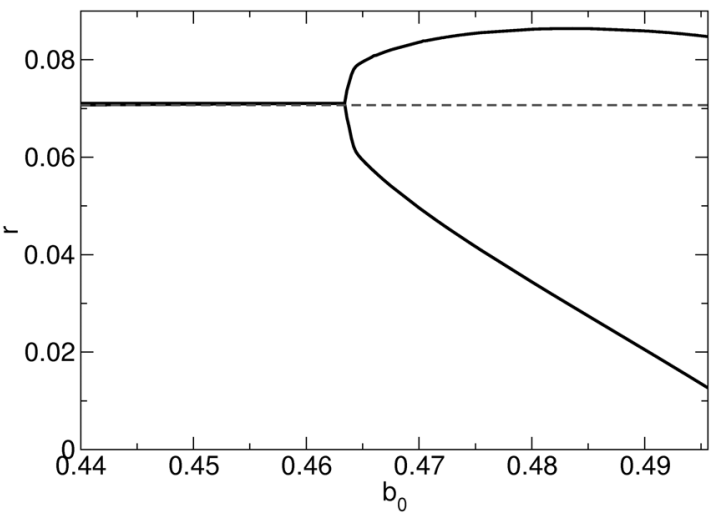

Fig. 2.4 Minimum and maximum radius (black), obtained from simulations, and radius of the Pyragas orbit $r=\sqrt{-\lambda}$ (dashed line) versus the control amplitude $b_{0}$. For large $b_{0}$ the stabilized Pyragas orbit becomes unstable in a torus (Neimark-Sacker) bifurcation (compare Fig. 2.2 (a), dashed line) $(\gamma=-10, \lambda=$ $\left.-0.005, \beta=\pi / 4, \tau=\frac{2 \pi}{1-\gamma \lambda}\right)$

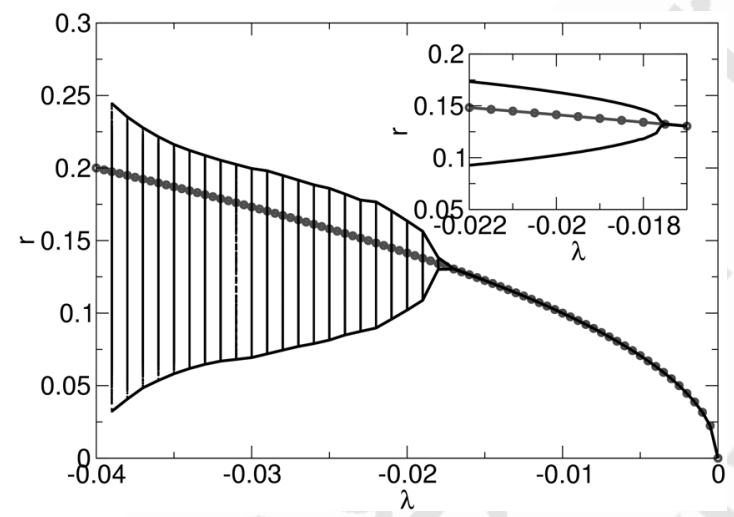

Fig. 2.5 Minimum and maximum radius (black), obtained from simulations, and radius of the Pyragas orbit $r=\sqrt{-\lambda}$ (circles) versus $\lambda$. For sufficiently negative $\lambda$ the stabilized Pyragas orbit becomes unstable in a torus (Neimark-Sacker) bifurcation. $(\gamma=$ $\left.-10, b_{0}=0.3, \beta=\pi / 4, \tau=\frac{2 \pi}{1-\gamma \lambda}\right)$

bifurcating 2-torus inherits stability, taking it away from the Pyragas orbit by exchange of stability.

Equivariance under rotations causes a slight improvement over standard torus bifurcation. The rotation number along the bifurcating torus branch, which would behave in a devil's staircase manner in the general case, in fact becomes a smooth function of the amplitude of the resulting oscillations of 
$r(t)=|z(t)|$. In particular, the usual phase locking or resonance phenomena at rational rotation numbers on invariant tori do not occur. This can be seen, for example, by an analysis in suitable Palais coordinates along the relative equilibrium to the $S^{1}$-action which is given by the destabilized Pyragas orbit. Eliminating the $S^{1}$-action, the Neimark-Sacker bifurcation then becomes a nondegenerate relative Hopf bifurcation from the relative equilibrium $\zeta_{0}=r_{0}$. The Hopf eigenvalues are provided by the purely imaginary Floquet exponent $\Lambda$. See Ref. [36] and the survey [37] for details of such a setting. A similar phenomenon occurs, albeit in a reaction-diffusion setting, when rigidly rotating spiral waves in excitable media destabilize to meandering motions $[37,38]$. For the likewise related destabilization of wavy vortices to modulated wavy vortices in Taylor-Couette fluid flow between rotating concentric cylinders see [39] and the references there. Intensity pulsations in laser systems provide yet another experimental source of the same phenomenon of smooth parameter dependence of rotation numbers (see Refs. [40,41]).

Unlike the present case, though, these phenomena do not involve delayed feedback control. In our case of Pyragas control we can conclude that stabilization and destabilization of Pyragas orbits occurs, either by transcritical bifurcations of non-Pyragas periodic orbits, or else by Neimark-Sacker torus bifurcation with nonresonant, smooth dependence of the rotation number.

\section{6}

\section{Conclusion}

In conclusion, we have refuted the claim that a periodic orbit with an odd number of real Floquet multipliers greater than unity cannot be stabilized by time-delayed feedback control of Pyragas type. For this purpose we have analyzed the generic example of the normal form of a subcritical Hopf bifurcation, which is paradigmatic for a large class of nonlinear systems. We have worked out explicit analytical conditions for stabilization of the periodic orbit generated by a subcritical Hopf bifurcation in terms of the amplitude and the phase of the feedback control gain. Our results emphasize the crucial role of a non-vanishing phase of the control signal for stabilization of periodic orbits which violate the odd-number limitation. The feedback phase is readily accessible and can be adjusted, for instance, in laser systems, where subcritical Hopf bifurcation scenarios are abundant and Pyragas control can be realized via coupling to an external Fabry-Perot resonator [18]. The importance of the feedback phase for the stabilization of steady states in lasers [18] and neural systems [42], as well as for stabilization of periodic orbits by a time-delayed feedback control scheme using spatio-temporal filtering [43], has been noted recently. Here, we have shown that the odd-number limitation does not hold in general, which opens up fundamental questions as well as a wide range of 
applications. The result will not only be important for practical applications in physical sciences, technology, and life sciences, where one might often desire to stabilize periodic orbits with an odd number of positive Floquet exponents. It also suggests noninvasive experimental and numerical tracking of unstable orbits and bifurcation analysis using time-delayed feedback control [44].

\section{Acknowledgements}

This work was supported by Deutsche Forschungsgemeinschaft in the framework of Sfb 555. We acknowledge helpful discussions with A. Amann, W. Just, and A. Pikovsky.

\section{Appendix: Calculation of Floquet Exponents}

The Floquet exponents $\Lambda$ of the Pyragas orbit can be calculated explicitly by rewriting Eq. (2.2) in polar coordinates $z(t)=r(t) e^{i \varphi(t)}$

$$
\begin{gathered}
\dot{r}(t)=\left(\lambda+r^{2}\right) r+b_{0}[\cos (\beta+\varphi(t-\tau)-\varphi) r(t-\tau)-r \cos \beta] \\
\dot{\varphi}(t)=1+\gamma r^{2}+b_{0}\left[\sin (\beta+\varphi(t-\tau)-\varphi) \frac{r(t-\tau)}{r}-\sin \beta\right]
\end{gathered}
$$

and linearizing around the periodic orbit according to $r(t)=r_{0}+\delta r(t)$ and $\varphi(t)=\Omega t+\delta \varphi(t)$, with $r_{0}=\sqrt{-\lambda}$ and $\Omega=1-\gamma \lambda$ (see Eq. (2.3)). This yields

$$
\begin{aligned}
\left(\begin{array}{c}
\delta \dot{r}(t) \\
\delta \dot{\varphi}(t)
\end{array}\right)= & {\left[\begin{array}{cc}
-2 \lambda-b_{0} \cos \beta & b_{0} r_{0} \sin \beta \\
2 \gamma r_{0}-b_{0} \sin \beta r_{0}^{-1} & -b_{0} \cos \beta
\end{array}\right]\left(\begin{array}{c}
\delta r(t) \\
\delta \varphi(t)
\end{array}\right) } \\
& +\left[\begin{array}{cc}
b_{0} \cos \beta & -b_{0} r_{0} \sin \beta \\
b_{0} \sin \beta r_{0}^{-1} & b_{0} \cos \beta
\end{array}\right]\left(\begin{array}{c}
\delta r(t-\tau) \\
\delta \varphi(t-\tau)
\end{array}\right) .
\end{aligned}
$$

With the ansatz

$$
\left(\begin{array}{c}
\delta r(t) \\
\delta \varphi(t)
\end{array}\right)=u \exp (\Lambda t)
$$

where $u$ is a two-dimensional vector, one obtains the autonomous linear equation

$$
\left[\begin{array}{cc}
-2 \lambda+b_{0} \cos \beta\left(e^{-\Lambda \tau}-1\right)-\Lambda & -b_{0} r_{0} \sin \beta\left(e^{-\Lambda \tau}-1\right) \\
2 \gamma r_{0}+b_{0} r_{0}^{-1} \sin \beta\left(e^{-\Lambda \tau}-1\right) & b_{0} \cos \beta\left(e^{-\Lambda \tau}-1\right)-\Lambda
\end{array}\right] u=0 .
$$


The condition of vanishing determinant then gives the transcendental characteristic equation

$$
\begin{aligned}
0 & =\left(-2 \lambda+b_{0} \cos \beta\left(e^{-\Lambda \tau}-1\right)-\Lambda\right)\left(b_{0} \cos \beta\left(e^{-\Lambda \tau}-1\right)-\Lambda\right) \\
& +b_{0} r_{0} \sin \beta\left(e^{-\Lambda \tau}-1\right)\left(2 \gamma r_{0}+b_{0} r_{0}^{-1} \sin \beta\left(e^{-\Lambda \tau}-1\right)\right)
\end{aligned}
$$

for the Floquet exponents $\Lambda$ which can be solved numerically.

\section{References}

1 E. Schöll and H. G. Schuster (Eds.): Handbook of Chaos Control (Wiley-VCH, Weinheim, 2007), second completely revised and enlarged edition.

2 E. Ott, C. Grebogi, and J. A. Yorke: Controlling chaos, Phys. Rev. Lett. 64, 1196 (1990).

3 S. Boccaletti, C. Grebogi, Y. C. Lai, H. Mancini, and D. Maza: The control of chaos: theory and applications, Phys. Rep. 329, 103 (2000).

4 D. J. Gauthier: Resource letter: Controlling chaos, Am. J. Phys. 71, 750 (2003).

5 K. Pyragas: Continuous control of chaos by self-controlling feedback, Phys. Lett. A 170, 421 (1992).

6 K. Pyragas and A. Tamaševičius: $E x$ perimental control of chaos by delayed selfcontrolling feedback, Phys. Lett. A 180, 99 (1993).

7 S. Bielawski, D. Derozier, and P. Glorieux: Controlling unstable periodic orbits by a delayed continuous feedback, Phys. Rev. E 49, R971 (1994).

8 T. Pierre, G. Bonhomme, and A. Atipo: Controlling the chaotic regime of nonlinear ionization waves using time-delay autosynchronisation method, Phys. Rev. Lett. 76, 2290 (1996).

9 K. Hall, D. J. Christini, M. Tremblay, J. J. Collins, L. Glass, and J. Billette: Dynamic control of cardiac alterans, Phys. Rev. Lett. 78, 4518 (1997).

10 D. W. Sukow, M. E. Bleich, D. J. Gauthier, and J. E. S. Socolar: Controlling chaos in a fast diode resonator using time-delay autosynchronisation: Experimental observations and theoretical analysis, Chaos 7, 560 (1997).
11 O. Lüthje, S. Wolff, and G. Pfister: Control of chaotic taylor-couette flow with time-delayed feedback, Phys. Rev. Lett. 86, 1745 (2001).

12 P. Parmananda, R. Madrigal, M. Rivera, L. Nyikos, I. Z. Kiss, and V. Gáspár: Stabilization of unstable steady states and periodic orbits in an electrochemical system using delayed-feedback control, Phys. Rev. E 59, 5266 (1999).

13 J. M. Krodkiewski and J. S. Faragher: Stabilization of motion of helicopter rotor blades using delayed feedback - modelling, computer simulation and experimental verification, J. Sound Vib. 234, 591 (2000).

14 T. Fukuyama, H. Shirahama, and Y. Kawai: Dynamical control of the chaotic state of the current-driven ion acoustic instability in a laboratory plasma using delayed feedback, Phys. Plasmas 9, 4525 (2002).

15 C. von Loewenich, H. Benner, and W. Just: Experimental relevance of global properties of time-delayed feedback control, Phys. Rev. Lett. 93, 174101 (2004).

16 M. G. Rosenblum and A. Pikovsky: Controlling synchronization in an ensemble of globally coupled oscillators, Phys. Rev. Lett. 92, 114102 (2004).

17 O. V. Popovych, C. Hauptmann, and P. A. Tass: Effective desynchronization by nonlinear delayed feedback, Phys. Rev. Lett. 94, 164102 (2005).

18 S. Schikora, P. Hövel, H. J. Wünsche, E. Schöll, and F. Henneberger: All-optical noninvasive control of unstable steady states in a semiconductor laser, Phys. Rev. Lett. 97, 213902 (2006). 
19 E. Schöll, J. Hizanidis, P. Hövel, and G. Stegemann: Pattern formation in semiconductors under the influence of time-delayed feedback control and noise, in Analysis and control of complex nonlinear processes in physics, chemistry and biology, edited by L. Schimansky-Geier, B. Fiedler, J. Kurths, and E. Schöll (World Scientific, Singapore, 2007), pp. 135-183.

20 W. Just, T. Bernard, M. Ostheimer, E. Reibold, and H. Benner: Mechanism of timedelayed feedback control, Phys. Rev. Lett. 78, 203 (1997)

$21 \mathrm{H}$. Nakajima: On analytical properties of delayed feedback control of chaos, Phys. Lett. A 232, 207 (1997).

22 H. Nakajima and Y. Ueda: Limitation of generalized delayed feedback control, Physica D 111, 143 (1998).

23 I. Harrington and J. E. S. Socolar: Limitation on stabilizing plane waves via time-delay feedback, Phys. Rev. E 64, 056206 (2001).

24 K. Pyragas, V. Pyragas, and H. Benner: Delayed feedback control of dynamical systems at subcritical Hopf bifurcation, Phys. Rev. E 70, 056222 (2004).

25 V. Pyragas and K. Pyragas: Delayed feedback control of the Lorenz system: An analytical treatment at a subcritical Hopf bifurcation, Phys. Rev. E 73, 036215 (2006)

26 J. E. S. Socolar, D. W. Sukow, and D. J. Gauthier: Stabilizing unstable periodic orbits in fast dynamical systems, Phys. Rev. E 50, 3245 (1994).

27 H. G. Schuster and M. B. Stemmler: Control of chaos by oscillating feedback, Phys. Rev. E 56, 6410 (1997).

28 H. Nakajima and Y. Ueda: Half-period delayed feedback control for dynamical systems with symmetries, Phys. Rev. E 58, 1757 (1998).

29 K. Pyragas: Control of chaos via an unstable delayed feedback controller, Phys. Rev. Lett. 86, 2265 (2001).

30 B. Fiedler, V. Flunkert, M. Georgi, P. Hövel, and E. Schöll: Refuting the odd number limitation of time-delayed feedback control, Phys. Rev. Lett. 98, 114101 (2007).

31 W. Just, B. Fiedler, V. Flunkert, M. Georgi, P. Hövel, and E. Schöll: Beyond odd number limitation: a bifurcation analysis of timedelayed feedback control, Phys. Rev. E (2007).
32 O. Diekmann, S. A. van Gils, S. M. Verduyn Lunel, and H. O. Walther: Delay Equations (Springer-Verlag, New York, 1995).

33 A. Amann (private communication).

34 B. Fiedler: Global Bifurcation of Periodic Solutions with Symmetry (Springer-Verlag, Heidelberg, 1988).

35 A. Vanderbauwhede: Centre manifolds, normal forms and elementary bifurcations, Dynamics Reported 2, 89 (1989).

36 B. Fiedler, B. Sandstede, A. Scheel, and C. Wulf: Bifurcation from Relative Equilibria of Noncompact Group Actions: Skew Products, Meanders, and Drifts, Documenta Mathematica 1, 479 (1996).

37 B. Fiedler and A. Scheel: Dynamics of reaction-diffusion patterns, in Trends in Nonlinear Analysis, Festschrift dedicated to Willi Jäger for his 60 th birthday, edited by M. Kirkilionis, R. Rannacher, and F. Tomi (Springer, Heidelberg, 2002).

38 B. Fiedler, M. Georgi, and N. Jangle: Spiral wave dynamics: Reaction and diffusion versus kinematics, in Analysis and Control of complex nonlinear Processes in Physics, Chemistry and Biology (World Scientific, Singapore, 2007).

39 M. Golubitsky and I. Stewart: Singularities and Groups in Bifurcation Theory. Volume 2, vol. 69 (Springer-Verlag, New York, 1988).

40 S. Bauer, O. Brox, J. Kreissl, B. Sartorius, M. Radziunas, J. Sieber, H. J. Wünsche, and F. Henneberger: Nonlinear dynamics of semiconductor lasers with active optical feedback, Phys. Rev. E 69, 016206 (2004).

41 S. Wieczorek, B. Krauskopf, and D. Lenstra: Unifying view of bifurcations in a semiconductor laser subject to optical injection, Optics Communications 172, 279 (1999).

42 M. G. Rosenblum and A. Pikovsky: Delayed feedback control of collective synchrony: An approach to suppression of pathological brain rhythms, Phys. Rev. E 70, 041904 (2004).

43 N. Baba, A. Amann, E. Schöll, and W. Just: Giant improvement of time-delayed feedback control by spatio-temporal filtering, Phys. Rev. Lett. 89, 074101 (2002).

44 J. Sieber and B. Krauskopf: Control based bifurcation analysis for experiments, Nonlinear Dynamics, DOI: 10.1007/s11071-007-9217-2 (2007) 


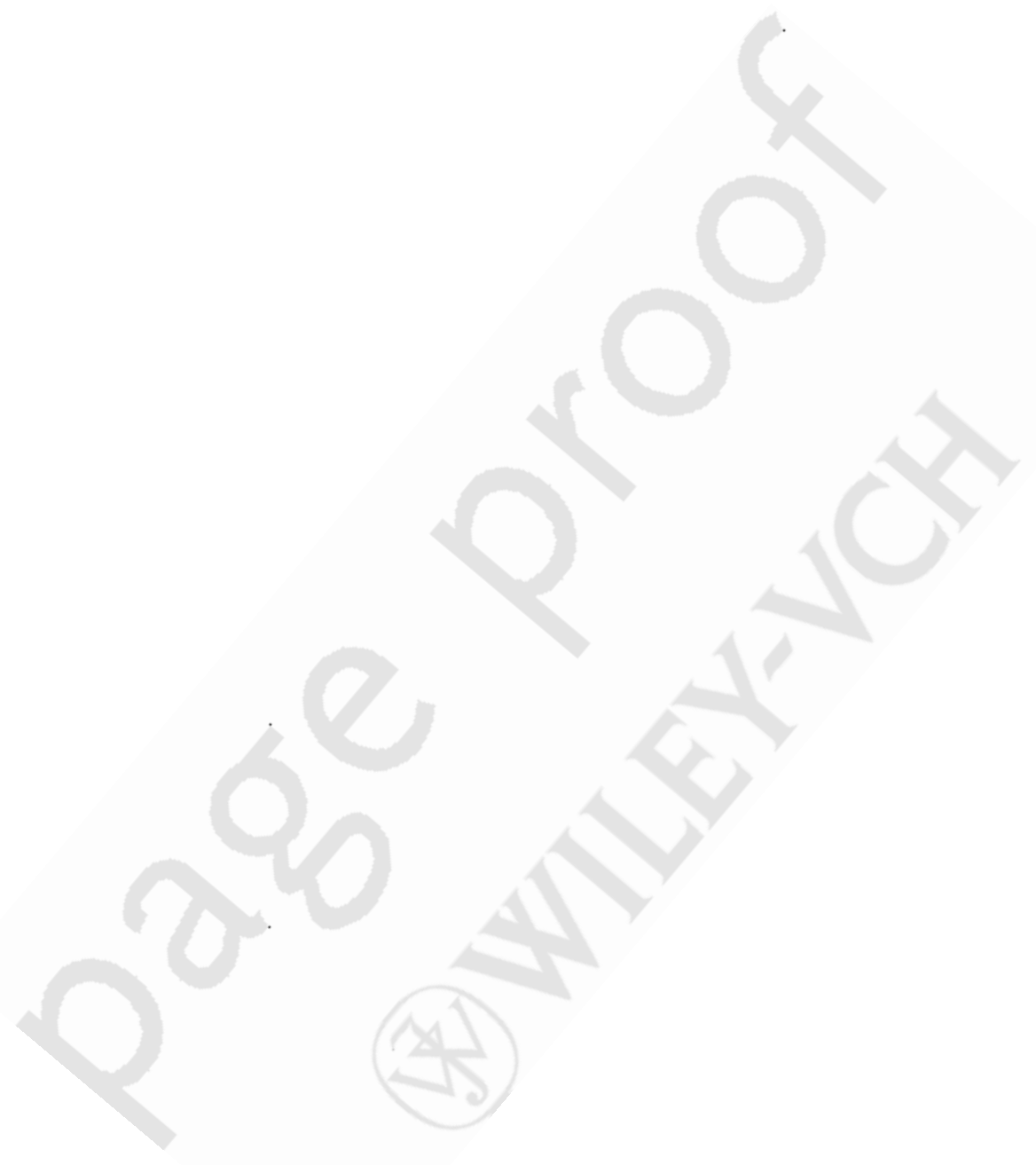

